\title{
Futures Hedges under Basis Heteroscedasticity
}

\author{
Subhankar Nayak and Jacques A. Schnabel \\ School of Business \& Economics, Wilfrid Laurier University, Waterloo, ON, Canada N2L 3C5 \\ Correspondence should be addressed to Jacques A. Schnabel, jschnabel@wlu.ca
}

Received 30 October 2012; Accepted 26 November 2012

Academic Editors: J. H. Haslag, T. Kuosmanen, and J. Zarnikau

Copyright () 2012 S. Nayak and J. A. Schnabel. This is an open access article distributed under the Creative Commons Attribution License, which permits unrestricted use, distribution, and reproduction in any medium, provided the original work is properly cited.

\begin{abstract}
Minimum variance and mean-variance optimizing hedges are developed when basis risk exhibits heteroscedasticity; that is, the variance of the difference between spot and futures prices is not constant but rises with the level of spot prices. Two different hedging objectives are modeled and optimized. The resulting optimality conditions are then interpreted both analytically and intuitively. Simulations are run to determine whether the model proposed here is superior to the traditional model in terms of minimizing the hedger's terminal wealth. The resulting hedge ratios are shown to differ from those that are obtained for the traditional homoscedastic basis case, but consistent with the extant theoretical paradigm, the demand for futures contacts is dichotomized into pure hedging and pure speculative components. The simulations demonstrate that, under the statistical assumptions invoked, the proposed model implies uniformly less hedging and a lower variance of terminal wealth compared with the traditional model.
\end{abstract}

\section{Introduction}

In the usual textbook treatment of the hedging decision, the basis or the difference between spot and futures prices is assumed to exhibit a constant variance. Examples are provided by Duffie [1], Hull [2], and Stulz [3]. The empirical research that has questioned the validity of this assumption of basis homoscedasticity has sought remediation by invoking sophisticated econometric techniques that do not entail the unchanging variance requirement, for example, the autoregressive conditional heteroscedastic (ARCH) model invoked by Park and Bera [4] and the generalized autoregressive conditional heteroscedastic $(\mathrm{GARCH})$ model proposed by Kalimipalli and Susmel [5].

This paper takes a different tack to the issue by examining how the hedging decision itself changes as a result of removing the restriction that the basis exhibits a fixed variance. Formulas for the minimum variance and the mean-variance optimizing hedges are developed when the variance of the basis is assumed to increase with the level of spot prices.

\section{Basis Heteroscedasticity}

This paper examines hedging in the traditional context of a single-period decision model where the hedge is formed at time 0 , and the hedge is terminated or lifted at time 1 . To provide a concrete decision context, the specific case of a firm wishing to hedge a foreign currency receivable amounting to $Q$ that is projected to be received at time 1 is considered. The foregoing is consistent with the empirical evidence provided by Grabbe [6], who argues that the basis for foreign currency contracts exhibits this type of heteroscedasticity.

Define $S_{0}$ and $S_{1}$ as the spot rates or the values of the foreign currency that prevail as the start and end, respectively, of the period. Employing direct quotation, all exchange rates considered in this paper are given as the number of domestic currency units per unit of the foreign currency examined. Similarly, define $F_{0}$ and $F_{1}$ as the futures rates that prevail at the start and end, respectively, of the period. Note that, whereas $S_{0}$ and $F_{0}$ are observed at the time the hedge is formed, $S_{1}$ and $F_{1}$ are unknown and thus are the random variables in this discussion. The existing literature on hedging conventionally assumes that the basis or $\left(S_{1}-F_{1}\right)$ exhibits an unchanging variance; that is, $\sigma^{2}\left(S_{1}-F_{1}\right)$ is a constant.

Instead of the foregoing, this paper conjectures the percentage basis or the basis calculated as a percent of the spot rate; that is, $e=\left(S_{1}-F_{1}\right) / S_{1}$ exhibits a constant variance. Stated another way, this paper assumes that the basis itself, $\left(S_{1}-F_{1}\right)$, exhibits a variance that rises with $S_{1}$. Thus, a 
heteroscedastic, rather than the traditional homoscedastic, basis is assumed.

Absent hedging, the firm's end-of-period wealth or $W$, equals $S_{1} Q$. Define $x$ as the firm's short position in futures contracts on the foreign currency; that is, the number of units of the foreign currency that the firm sells via futures contracts at time 0 . This short futures hedge is terminated at time 1 , the end of the single time period considered here. Thus, the firm's hedged terminal wealth, $W$, equals $S_{1} Q+$ $\left(F_{0}-F_{1}\right) x$. Substituting the following equation

$$
F_{1}=S_{1}(1-e)
$$

into the expression for the firm's hedged terminal wealth results in the following alternative articulation of $W$ :

$$
W=S_{1}(Q-x)+e S_{1} x+F_{0} x .
$$

The extant hedging literature assumes, consistent with corroborating empirical evidence, that the expected value of the basis equals zero. By the same token, the assumption made here is the expected value of the basis calculated as a percent of the spot rate, or $e$ likewise equals zero. A further assumption invoked here, consistent with the evidence for interest rate futures provided by Park and Bera [4], is the statistical independence of $e$ and $S_{1}$.

Given the foregoing, basis risk or the variance of $\left(S_{1}-\right.$ $\left.F_{1}\right)$ equals $\sigma^{2}\left(e S_{1}\right)$. The statistical independence of $e$ and $S_{1}$ implies that this basis risk simplifies to the expression $\sigma^{2}(e) E\left(S_{1}^{2}\right)$. In contrast to basis risk, the expression $\sigma^{2}\left(S_{1}\right)$ denotes spot rate risk. It is well known that hedging with futures contracts involves the substitution of basis risk for spot rate risk, an interpretation that will be exploited later in this paper.

\section{Minimum Variance Hedges}

In the appendix, it is shown that the variance of the firm's terminal wealth simplifies to the following expression given the statistical assumptions that the expectation of $e$ and its variance are zero and constant, respectively, and that $e$ and $S_{1}$ are stochastically independent:

$$
\sigma^{2}(W)=(Q-x)^{2} \sigma^{2}\left(S_{1}\right)+x^{2} \sigma^{2}(e) E\left(S_{1}^{2}\right) .
$$

To determine the minimum variance hedge, the derivative of (3) with respect to $x$ is evaluated and equated to zero. This results in the following expression for $x^{*}$, the amount of futures contracts on the foreign currency that must be sold to effect a hedge which minimizes terminal wealth:

$$
x^{*}=Q\left[\frac{\sigma^{2}\left(S_{1}\right)}{\sigma^{2}\left(S_{1}\right)+\sigma^{2}(e) E\left(S_{1}^{2}\right)}\right] .
$$

The quotient $x^{*} / Q$ is known as the hedge ratio and denotes the fraction of the firm's exposure that is hedged. The denominator in the brackets in (4) may be interpreted as the total risk present in the hedging situation, defined as the sum of spot rate risk, $\sigma^{2}\left(S_{1}\right)$, and basis risk $\sigma^{2}\left(S_{1}-F\right)$ or $\sigma^{2}(e) E\left(S_{1}^{2}\right)$. Given these definitions, (4) provides an intuitive interpretation, which is articulated as follows.
The wealth-variance minimizing hedge ratio equals the fraction that spot rate risk comprises of the total risk present in the hedging situation. Since hedging may be interpreted as the process of substituting basis risk for spot rate risk, the common sense that underlies (4) is clear. In the absence of basis risk, the hedge ratio equals $100 \%$; that is, all the risk is hedged, whereas if basis risk is present, the hedge ratio is less than $100 \%$; that is, only a fraction of the risk is hedged as basis risk persists after the hedge is executed.

Duffie [1] identifies the variance minimizing solution of the firm's hedging problem as expressing the firm's pure hedging demand for futures contracts. Observing this terminology, (4) captures the firm's demand for futures contracts solely as a hedging instrument when basis risk is heteroscedastic.

The hedge ratio or $x^{*} / Q$ under basis heteroscedasticity, formulated as the bracketed term on the RHS (right-hand side) of (4), may be contrasted with the traditional hedge ratio under basis homoscedasticity derived in Duffie [1] or Hull [2], to wit

$$
\frac{x^{*}}{Q}=\frac{\operatorname{cov}\left(S_{1}, F\right)}{\sigma^{2}(F)}=\frac{\operatorname{cov}\left(S_{1}, S_{1}(1-e)\right)}{\sigma^{2}\left(S_{1}(1-e)\right)} .
$$

The second formula on the RHS of (5) is derived by adopting the traditional hedge ratio to the paradigm of this paper where $F=S_{1}(1-e)$. Clearly, the hedging decision is impacted by the basis behavior assumption invoked, as captured by these two contrasting formulas.

\section{Mean-Variance Optimizing Hedges}

In contrast to the preceding, this section posits the following objective function that the firm wishes to maximize:

$$
E(W)-\frac{\Theta}{2} \sigma^{2}(W)
$$

The parameter $\Theta$ measures the firm's degree of risk aversion, with high values depicting greater aversion to risk. The preceding section may be interpreted as one where this parameter approaches a value of infinity, that is, one of extreme risk aversion.

Calculating the expected value of (2) and then taking the derivative of the resulting expression with respect to $x$ implies the following:

$$
\frac{d E(W)}{d x}=F_{0}-E\left(S_{1}\right)
$$

To maximize expression (7), its derivative with respect to $x$ is set equal to zero. This results in the following requirement:

$$
F_{0}-E\left(S_{1}\right)-\Theta\left[x E\left(S_{1}^{2}\right) \sigma^{2}(e)-(Q-x) \sigma^{2}\left(S_{1}\right)\right]=0 .
$$

Equation (8) simplifies to the following optimal solution or $x^{* *}$, the amount of foreign currency futures that must be sold to effect a mean-variance optimizing hedge:

$$
\begin{aligned}
x^{* *}= & Q\left\{\frac{\sigma^{2}\left(S_{1}\right)}{\left[\sigma^{2}\left(S_{1}\right)+\sigma^{2}(e) E\left(S_{1}^{2}\right)\right]}\right\} \\
& +\frac{F_{0}-E\left(S_{1}\right)}{\Theta\left[\sigma^{2}\left(S_{1}\right)+\sigma^{2}(e) E\left(S_{1}^{2}\right)\right]} .
\end{aligned}
$$


Recalling (4), the first component of (9) refers to the pure hedging demand for futures contracts. Again invoking Duffie's [1] terminology, the second component of (9) refers to the pure speculative demand for futures contracts. The numerator of this second component measures the expected profit from initially selling the futures contract at price $F_{0}$ and then buying back the same futures contract when the hedge is terminated at an expected price of $E\left(S_{1}\right)$. This expected profit is divided by the risk aversion parameter. Risk aversion attenuates the firm's speculative desire to capture this expected profit as greater aversion to risk implies that the firm in question places less weight on this expected profit. This expected profit is likewise divided by the total risk of the hedging situation, that is, the sum of spot rate risk and basis risk as discussed above. Thus, the presence of total risk in the hedging situation likewise serves to diminish the firm's speculative demand for futures contracts.

Equation (9) may be contrasted with the traditional formula obtained under basis homoscedasticity, as developed in Duffie [1], namely,

$$
x^{* *}=Q\left\{\frac{\operatorname{cov}\left(S_{1}, F\right)}{\sigma^{2}(F)}\right\}+\frac{F_{0}-E\left(S_{1}\right)}{\Theta\left[\sigma^{2}(F)\right]} .
$$

Translated into the paradigm of this paper where $F=S_{1}(1-$ $e),(10)$, may be restated as the following

$$
x^{* *}=Q\left\{\frac{\operatorname{cov}\left(S_{1}, S_{1}(1-e)\right)}{\sigma^{2}\left(S_{1}(1-e)\right)}\right\}+\frac{F_{0}-E\left(S_{1}\right)}{\Theta\left[\sigma^{2}\left(S_{1}(1-e)\right)\right]} .
$$

Observe that, while the dichotomy between pure hedging demand and pure speculative demand is obtained in both basis scenarios, that is, homo- versus heteroscedasticity, the specific formulas for these two demand components change, as evidenced by formulas (9) and (11).

\section{Simulations}

With a view to gauging whether the hedging paradigm developed here is superior to the traditional paradigm under the statistical assumptions invoked, simulations were conducted. To obviate the need to specify the numerical value of the subjective risk aversion parameter, $\Theta$, employed in Section 4, only the formulas derived in Section 3 are considered. Specifically, the novel hedge ratio proposed in (4) is compared with the traditional hedge ratio given in (5) both in terms of their numerical values as well as in terms of what these different hedge ratios imply about the resulting variance of the hedger's terminal wealth, as captured by (3).

For all the simulations, $Q$, the amount of foreign currency receivable, is set equal to unity for convenience. Thus, (4) and (5) yield the amount of the hedge for the proposed model and the traditional model, respectively, for a notional one unit of exposure. $S_{1}$, the uncertain spot exchange rate, is assumed to be normally distributed with an expected value of $\mu_{S}$ and a standard deviation of $\sigma_{S}$.

The traditional model specifies that the basis, $\varepsilon=S_{1}-F$, exhibits a constant variance. This basis is assumed to be normally distributed with an expected value of $\mu_{\varepsilon}$ and a standard deviation of $\sigma_{\varepsilon}$. In contrast, the model proposed in this paper assumes that $e$, the basis measured as a percent of the spot price, that is, $\left(S_{1}-F\right) / S_{1}$, exhibits a constant variance, is normally distributed with an expected value of $\mu_{e}$ and a standard deviation of $\sigma_{e}$, and is stochastically independent of $S_{1}$.

Five different sets of $S_{1}$ are generated, with mean values equaling 1.0, 2.0, 3.0, 4.0, and 5.0 and standard deviation values equaling $1.0,1.5,2.0,2.5$, and 3.0 , respectively. Likewise, five different sets of $e$ are generated, which specify that the mean value of $e$ equals zero, that the standard deviation of $e$ equals the values $0.5,1.0,1.5,2.0$, and 2.5, and that $e$ and $S_{1}$ are stochastically independent. Finally, five different sets of $\varepsilon$ are generated, with the mean value of $\varepsilon$ equaling zero and its standard deviation taking on the values of $0.5,1.0,1.5,2.0$, and 2.5 .

The foregoing combinations of statistical scenarios result in a total of 25 distinct simulation cases, which are listed as the rows in Table 1. Each scenario is simulated with 100,000 observations. Consistent with the assumption of stochastic independence invoked earlier, the correlation coefficients reported in the 9th and 10th columns are all close to zero. It is noteworthy that all the entries in the table's penultimate column are positive. These entries are the differences in the hedge ratio values obtained by employing the traditional model, which are reported in the 13th column, versus the hedge ratio values obtained by utilizing the model developed here, which are reported in the 11th column. The positivity of all the entries means that the proposed model implies less hedging compared to the traditional model. Thus, if the traditional hedging paradigm, derived under basis homoscedasticity, is applied to the heteroscedastic basis setting envisioned in this paper, excessive hedging ensues.

The most significant results of the simulations are found in the table's last column. The entries of that column equal the variance of the hedger's terminal wealth induced by employing the traditional model, that is, (5) which is reported in the 14th column, minus the same variance induced by employing the model proposed in this paper, that is, (4) which is reported in the 12th column. It is striking that all the entries in the last column are positive; that is, the model proposed here is superior to the traditional model as the variance of the hedger's terminal wealth is reduced by employing the former rather than the latter. The excessive hedging induced by the use of the traditional hedging formula results in higher variances of hedged terminal wealth.

\section{Conclusion}

This paper has reexamined the classic single-period model of hedging with futures contracts given mean-variance preferences under the novel assumption that basis risk is heteroscedastic, which contrasts with the traditional assumption of basis risk homoscedasticity. Both minimum variance and mean-variance optimizing objectives were entertained. Consistent with the paradigm developed for the homoscedastic basis risk case, the demand for futures contracts was dichotomized into two factors, namely, pure hedging and pure speculative components. The formulas developed for these two futures demand elements were 


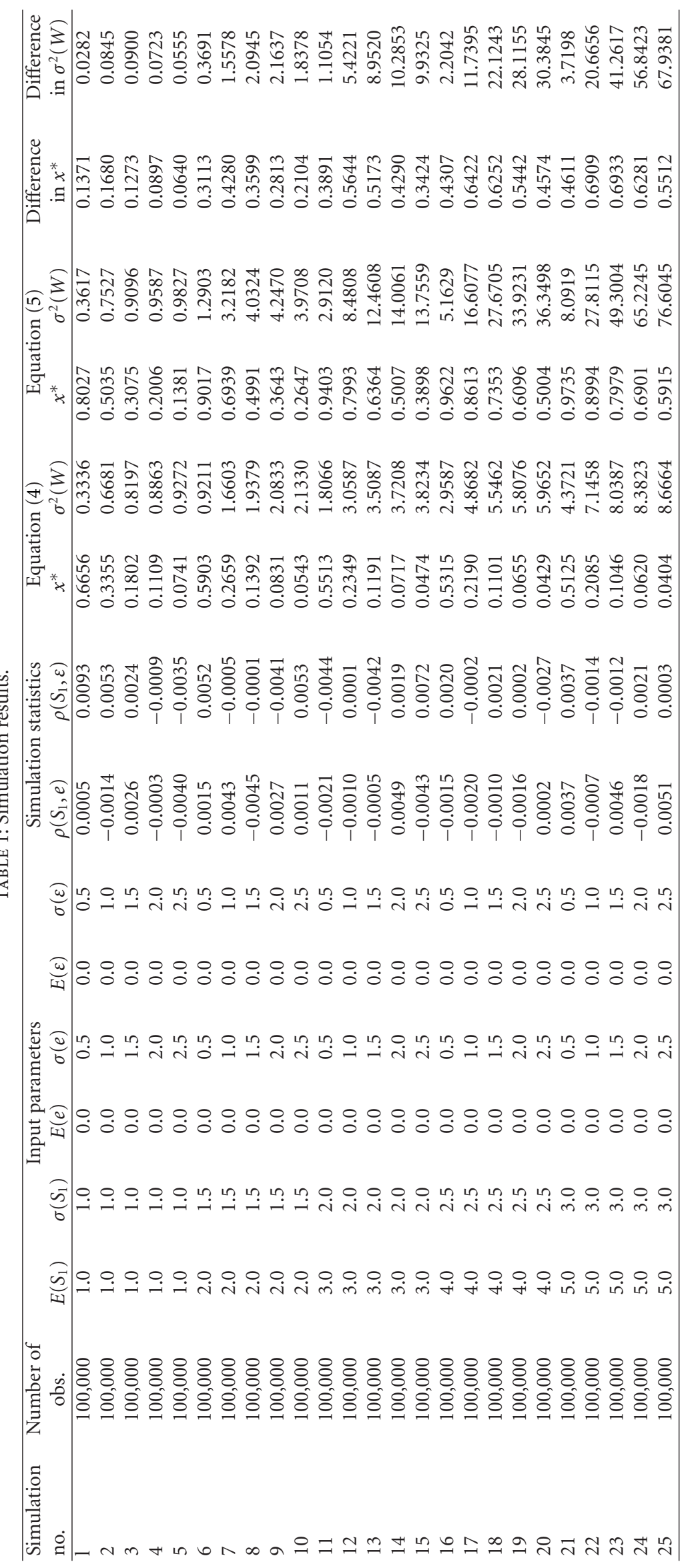


shown to accord with intuition. For example, the pure hedging demand is shown to be equal to the fraction of total risk, which is comprised of the sum of spot price risk and basis risk that is derived from spot price risk. Since hedging may be viewed as the substitution of basis risk for spot price risk, the common sense of the derived result is clear.

The specific formulas for these two demand components under the disparate assumptions of basis hetero- versus homoscedasticity were contrasted. Simulations were conducted to compare the performance of the model proposed here with the traditional model, under the statistical assumptions invoked; namely, the basis measured as a percent of the spot price exhibits a zero mean and a constant variance and is stochastically independent of the spot price. The simulations demonstrate that the proposed model implies uniformly less hedging and lower variances of hedged terminal wealth compared with the traditional model. Articulated another way, when the traditional model is applied in the statistical setting of this paper, excessive hedging occurs, which leads in turn to higher variances of hedged terminal wealth. These observations buttress the conclusion that the hedges developed in this paper are superior when basis heteroscedasticity prevails.

\section{Appendix}

This appendix proves that the variance of the hedger's terminal wealth is given by (3).

Bearing in mind (2), which delineates the components of the hedger's terminal wealth, and arguing from first principles, the variance of terminal wealth is given by the following equation:

$$
\begin{aligned}
\sigma^{2}(W)= & (Q-x)^{2} \sigma^{2}\left(S_{1}\right)+x^{2} \sigma^{2}\left(S_{1} e\right) \\
& +2(Q-x) x \operatorname{cov}\left(S_{1}, S_{1} e\right)
\end{aligned}
$$

Thus, to prove the validity of (3), the following two assertions must be established:

$$
\begin{gathered}
\operatorname{cov}\left(S_{1}, S_{1} e\right)=0, \\
\sigma^{2}\left(S_{1} e\right)=\sigma^{2}(e) E\left(S_{1}^{2}\right) .
\end{gathered}
$$

In the proof of these two assertions, a critical statistical theorem cited by Ingersoll [7, page 15] is repeatedly invoked; namely, two random variables $X$ and $Y$ are stochastically independent if and only if $\operatorname{cov}[f(X), g(X)]=0$ for all pairs of functions $f(\cdot)$ and $g(\cdot)$.

To prove (A.2), first observe that $\operatorname{cov}\left(S_{1}, e S_{1}\right)=E\left(S_{1}^{2} e\right)$ since $E(e)=0$. Via Ingersoll's cited theorem, $\operatorname{cov}\left(S_{1}^{2}, e\right)=0$. Thus, $E\left(S_{1}^{2} e\right)=0$ and therefore (A.2) is obtained.

To prove (A.3), first observe that $\sigma^{2}\left(S_{1} e\right)=E\left(S_{1}^{2} e^{2}\right)$ since $E(e)=0$. Via Ingersoll's cited theorem, $\operatorname{cov}\left(S_{1}^{2}, e^{2}\right)=0$. The foregoing implies that $E\left(S_{1}^{2} e^{2}\right)=E\left(S_{1}^{2}\right) E\left(e^{2}\right)$. Since $E\left(e^{2}\right)=$ $\sigma^{2}(e)$ because $E(e)=0$, (A.3) holds.

\section{References}

[1] D. Duffie, Futures Markets, Prentice Hall, Englewood Cliffs, NJ, USA, 1989.
[2] J. C. Hull, Fundamentals of Futures and Options Markets, Prentice Hall, Englewood Cliffs, NJ, USA, 7th edition, 2011.

[3] R. M. Stulz, Risk Management and Derivatives, Thomson SouthWestern, Mason, Ohio, USA, 2003.

[4] H. Y. Park and A. K. Bera, "Interest-rate volatility, basis risk and heteroscedasticity in hedging mortgages," Real Estate Economics, vol. 15, no. 2, pp. 79-97, 1987.

[5] M. Kalimipalli and R. Susmel, "Regime-switching stochastic volatility and short-term interest rates," Journal of Empirical Finance, vol. 11, no. 3, pp. 309-329, 2004.

[6] J. O. Grabbe, International Financial Markets, Prentice Hall, Englewood Cliffs, NJ, USA, 3rd edition, 1996.

[7] J. E. Ingersoll, Theory of Financial Decision Making, Rowman \& Littlefield, Totowa, NJ, USA, 1987. 


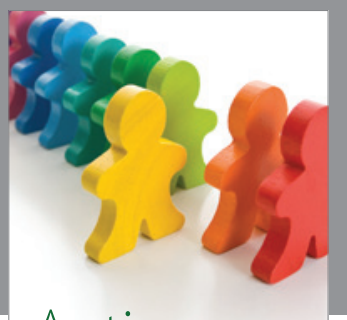

Autism

Research and Treatment
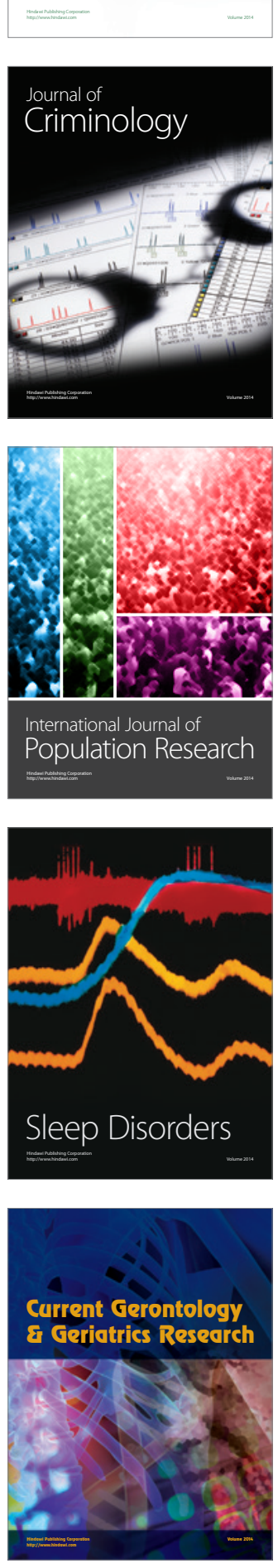
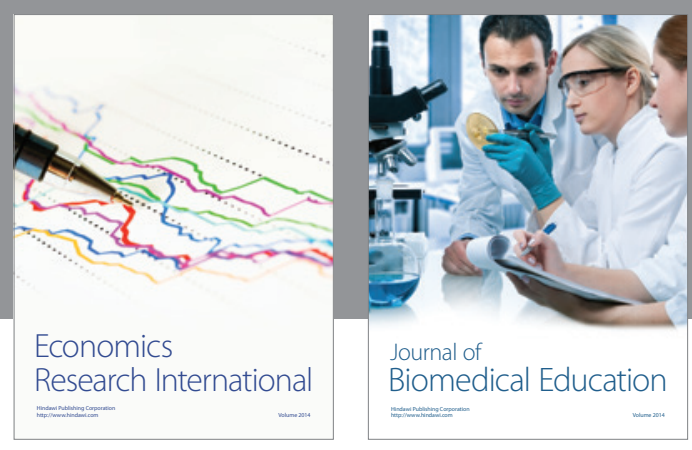

Journal of

Biomedical Education

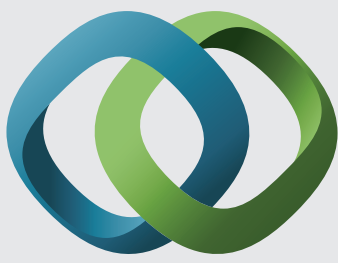

\section{Hindawi}

Submit your manuscripts at

http://www.hindawi.com
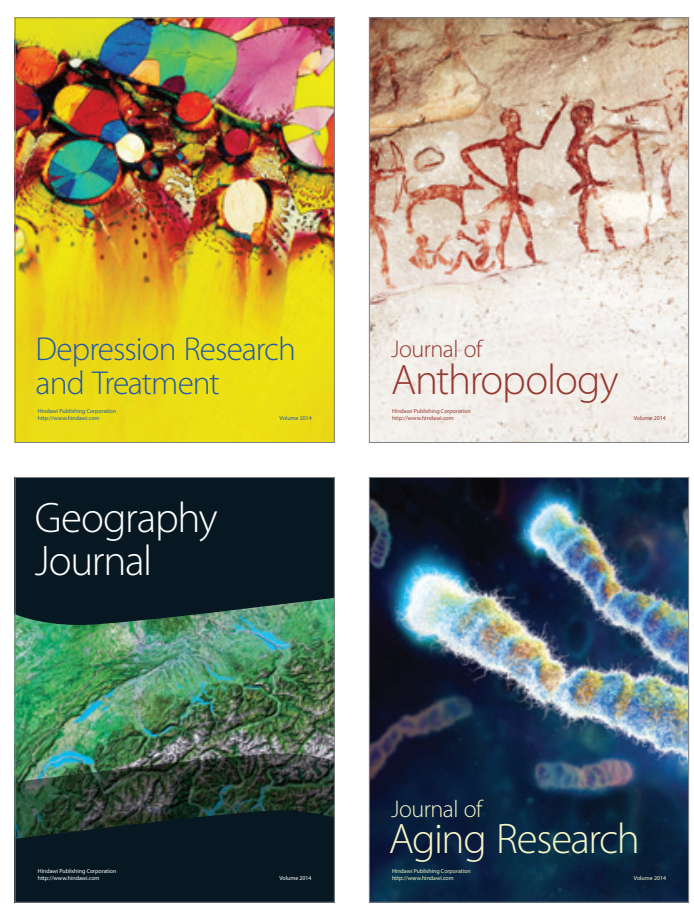

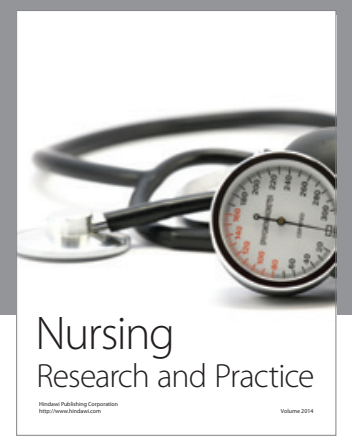

Nursing

Research and Practice

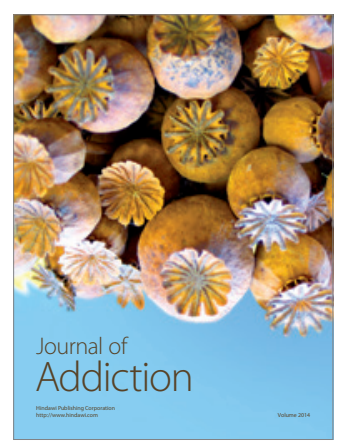

Child Development

Research

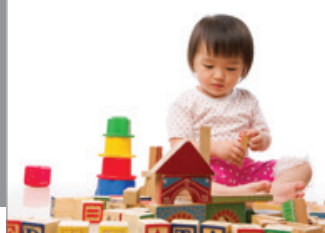

迥
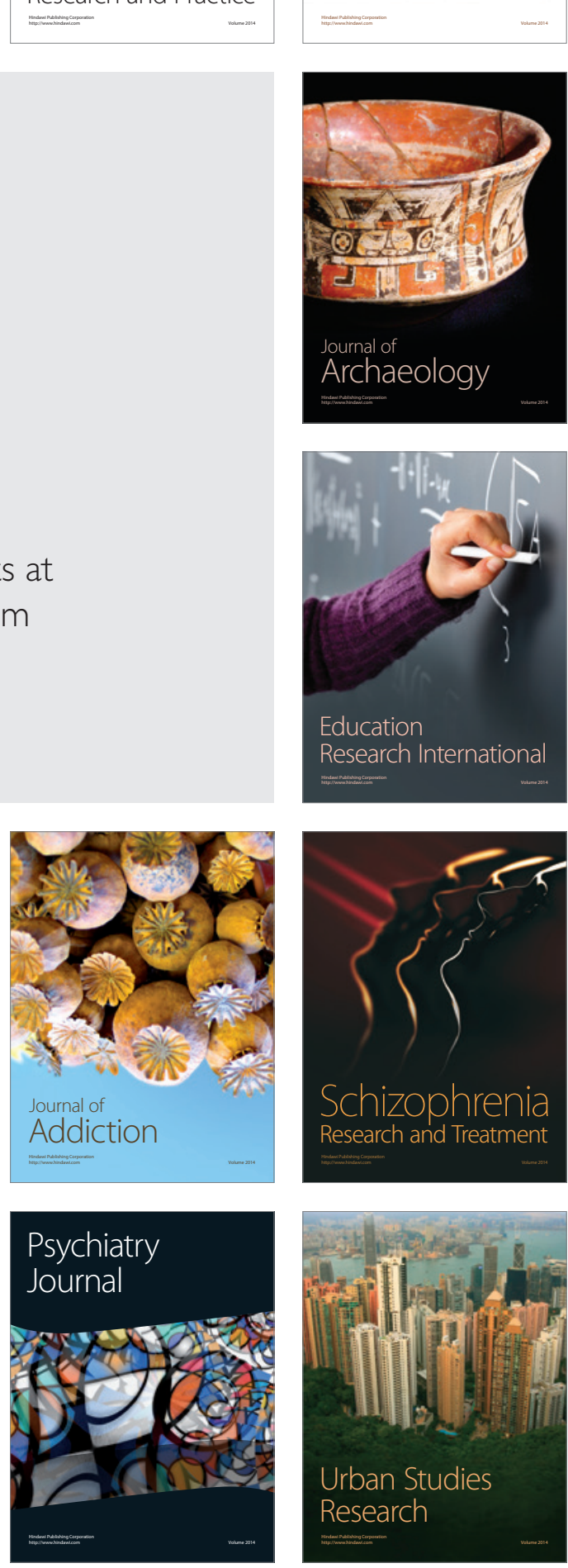